\title{
Photonic bandgap infrared spectrometer
}

\author{
H. Esat Kondakci, ${ }^{1,2}$ Mecit Yaman, ${ }^{1}$ Aykutlu Dana, ${ }^{1}$ and Mehmet Bayindir ${ }^{1,2, *}$ \\ ${ }^{1}$ UNAM-Institute of Materials Science and Nanotechnology, Bilkent University, 06800 Ankara, Turkey \\ ${ }^{2}$ Department of Physics, Bilkent University, 06800 Ankara, Turkey \\ ${ }^{*}$ Corresponding author: bayindir@nano.org.tr
}

Received 22 December 2009; revised 7 May 2010; accepted 19 May 2010; posted 28 May 2010 (Doc. ID 121671); published 21 June 2010

\begin{abstract}
We propose and demonstrate an infrared (IR) absorption spectrometer, made with a spatially variable photonic bandgap (PBG) structure, a blackbody source, and a simple IR detector, to identify the IR molecular fingerprints of analyte molecules. The PBG-based structure consists of thermally evaporated, IR transparent, high-refractive-index chalcogenide quarter-wave stacks (QWS) with a cavity layer. Spatial variation of the very sharp transmission peak due to the QWS cavity mode allows the structure to be used as a variable IR filter. Our proposed IR-PBG spectrometer can be used for detection and identification of volatile organic compounds. (C) 2010 Optical Society of America
\end{abstract}

OCIS codes: $\quad 230.5298,230.5750,120.6200,130.3060$.

\section{Introduction}

In infrared (IR) absorption spectroscopy, the molecular fingerprint of an analyte is obtained by passing an IR beam through the sample while observing the resulting characteristic absorption spectrum. Because only an incoherent blackbody source can be used to cover the whole IR spectrum, the spectral resolution of the absorption peaks are obtained either by a Fourier transform IR (FTIR) spectrometer, by carrying out direct spectral measurements using dispersive techniques, or via filter spectrometers designed specifically for a single wavelength that operate as on/off chemical sensors. In this paper, we introduce an IR absorption spectrometer based on a spatially/spectrally variable filter [1] that, unlike fixed filter spectrometers, enables concurrent measurement of any spectrally relevant IR spectrum. The photonic bandgap (PBG) filter is based on a spatially varying $\mathrm{PBG}$ multilayer fabricated from chalcogenide quarter-wave stacks [2,3]. Chalcogenide glasses $[4,5]$ are excellent materials to be used in IR devices because chalcogen elements ( $\mathrm{S}, \mathrm{Se}$, and $\mathrm{Te}$ ) make stable IR transmitting glasses, such as As-S, As-Se and Ge-As-Se-Te (GAST), that can be readily deposited using thermal evaporation. Therefore,

0003-6935/10/183596-05\$15.00/0

(C) 2010 Optical Society of America high refractive index contrast chalcogenides are being extensively used in PBG mirrors [6,7], filters $[1,8]$, fibers [9], and in chemical and biological sensors $[\underline{10}, \underline{11}]$.

\section{Spectrometer Design and Numerical Simulation}

The IR-PBG spectrometer consists of a spatially/ spectrally variable chalcogenide PBG filter, a hot filament as a blackbody source, and a simple IR detector or bolometer array that measures the intensity of the incident IR beam. The PBG structure can be fabricated to filter any wavelength along its length by using slanted evaporation geometry [1]. Two alternative IR-PBG spectrometer designs are suggested, as shown in Fig. 1. In the first design, the blackbody radiation is collimated using a pinhole, passed through a chamber containing the analyte, and is spectrally filtered by the movable IR-PBG structure. Finally, an integrative IR detector measures the total integrated intensity of the incoming electromagnetic (EM) wave [Fig. 1(a)]. Alternatively, as a second design, a concave mirror can be used to obtain a paraxial IR beam and an array of bolometers is used to measure intensity simultaneously with respect to filter position corresponding to a specific wavelength [Fig. 1(b)].

First, the working principle of the IR-PBG spectrometer will be described using a bolometer-arraybased design since it is easier to demonstrate the 
(a)

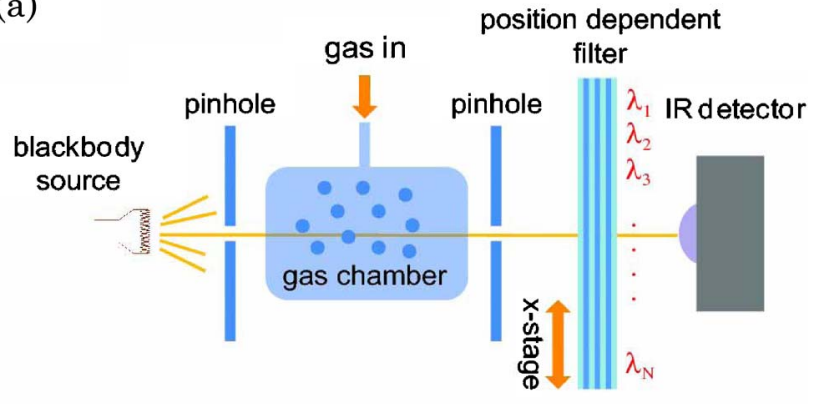

(b)

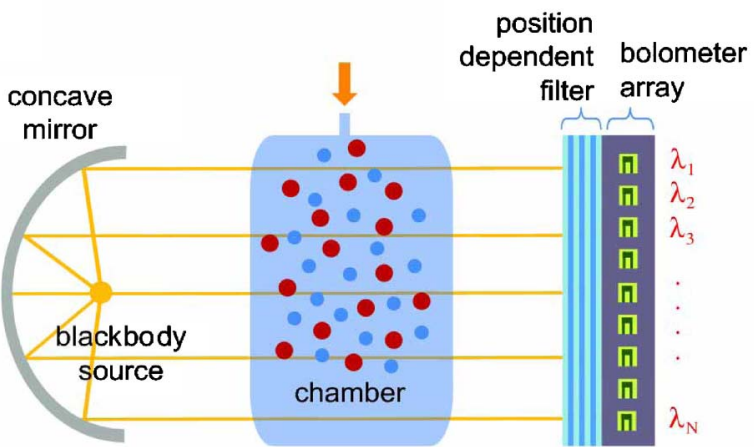

Fig. 1. (Color online) Proposed schemes for IR absorption spectrometry using spatially variable PBG filter. (a) IR-PBG spectrometer design used in the present work, in which electromagnetic radiation is spectrally filtered by a PBG filter and detected by an integrative IR detector for each position of the spatially variable filter. The detector signal abruptly decreases when the molecular absorption line coincides with the cavity mode corresponding to the position of the filter. (b) As an alternative design, a concave mirror can be used to collimate the IR beam and the bolometer array concurrently measures each intensity corresponding to a different position of the filter, effectively removing the movable components.

utility of the variable PBG filter in the static configuration. Low-cost bolometer arrays are available for IR imaging [12], which further makes this design favorable. The bolometer array is arranged parallel to the variable filter so that each bolometer only measures a spectrally filtered EM radiation. In the absence of an analyte, each bolometer will record a background intensity corresponding to its spatial position with respect to the filter. In the presence of an analyte, the recorded signal of each bolometer will be multiplied by the corresponding spectroscopic transmittance of the analyte molecules. Therefore, any change in a bolometer signal will be due to an absorption peak of the analyte. In this way, the whole absorption spectrum of the analyte molecules can be obtained simultaneously and restricted either by the spatial resolution of the bolometer array or the cavity mode bandwidth.

On the other hand, the movable-filter-based design can overcome this spatial resolution restriction by introducing a mechanical data collection step. In this design a single IR detector, sensitive in the range of the measurement, is used behind the IR filter. The selected wavelength is filtered by shifting the variable filter with respect to the pinhole and the detec- tor. After a background scan is taken without the analyte for each position of the filter (i.e., for each wavelength), the analyte is inserted and a second scan is taken. The position-dependent intensity difference can be obtained from

$$
I(x)=\int_{\lambda_{L}}^{\lambda_{U}} I_{S}(\lambda) T_{\mathrm{PBG}}(\lambda, x) D(\lambda)\left[T_{A}(\lambda)-1\right] \mathrm{d} \lambda,
$$

where $I_{S}(\lambda)$ is the spectral intensity of a blackbody, $T_{\mathrm{PBG}}(\lambda, x)$ is the transmission spectrum of the PBG filter at position $x, T_{A}(\lambda)$ is the transmission spectrum of the analyte, $D(\lambda)$ is the frequency response of the IR detector, and $\lambda_{L}$ and $\lambda_{U}$ are the lower and upper detection limits of the IR detector. While the filter is shifted along its axis behind the pinhole, the total integrated intensity will change abruptly when the filtered wavelength coincides with the absorption peak of the analyte. In effect, the same abrupt change in the integrated intensity, in principle, can be obtained without a background scan.

The PBG structure with $7+8$ quarter-wave stacks of $\mathrm{As}_{2} \mathrm{~S}_{3}$ /GAST system is simulated using the transfer matrix method (TMM). A blackbody source with an emission peak at $4.5 \mu \mathrm{m}(650 \mathrm{~K})$ is selected as the IR source. The PBG structure with $7+8$ quarterwave stacks of an $\mathrm{As}_{2} \mathrm{~S}_{3}$ GAST system is simulated using an incoherent layer transfer matrix method (TMM). The calculated transmittance, $I_{S}(\lambda) T_{\mathrm{PBG}}$ $(\lambda, x) T_{A}(\lambda)$, and the intensity difference obtained from Eq. (1) are shown in Figs. 2(a) and 2(b) as a function of position. The absorption line can be clearly resolved, which enables the molecular detection of the analyte [Figs. 2(b) and 2(c)]. In this simulation, a flat frequency response is used for the IR detector.

The resolution of the spectrometer depends on multiple design parameters. In the second design, the bandwidth of the passband $(30-60 \mathrm{~nm})$ is the limiting factor. In an ideal design that uses a larger number of layers in the PBG structure, the width of the transmission band can be further reduced. The thickness gradient, along with the resolution bandwidth, determines the minimum separation between bolometer-array elements. In our case, an ideal array element spacing of about $500 \mu \mathrm{m}$ is calculated for optimal resolution. In principle, the spacing can be made close to the wavelength, reducing the overall size of the spectrometer. The sensitivity of the system depends on further design parameters. Overall sensitivity is a function of the path length between the IR source and the sensor array, as well as the noise properties of the bolometers. In principle, the sensitivity can be improved using a multipass cavity and a bolometer array with a high dynamic range and low noise floor. An important feature of the design is simultaneous detection of multiple wavelengths using a bolometer array, resulting in the rejection of nonspecific effects, such as short- and long-term fluctuations in the light source intensity or environmental temperature fluctuations. 

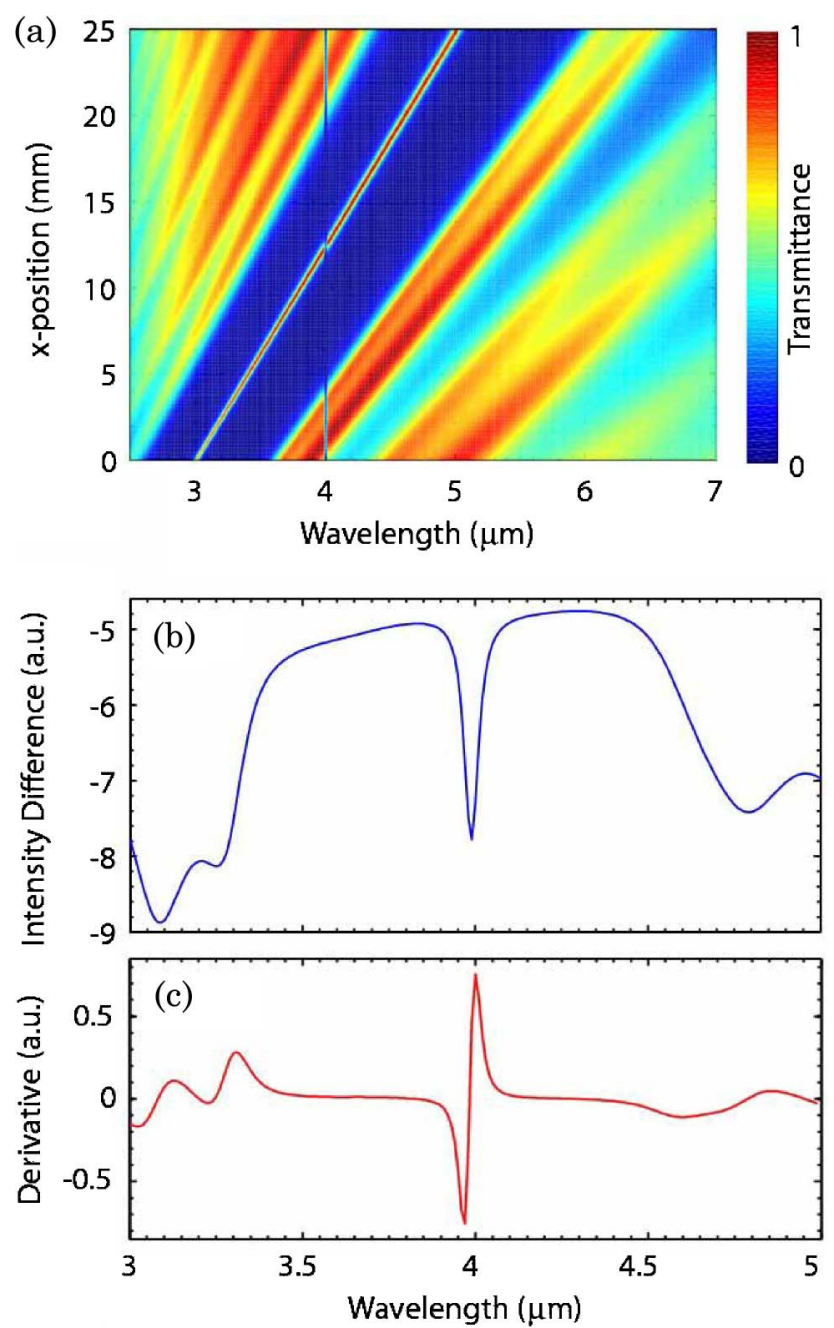

Fig. 2. (Color online) (a) Simulated transmittance spectrum through IR-PBG filter with a cavity mode shifting from 3 to $5 \mu \mathrm{m}$ within $25 \mathrm{~mm}$, in the presence of a hypothetical chemical that absorbs $95 \%$ of the incoming radiation at $4 \mu \mathrm{m}$ (FWHM $16 \mathrm{~nm}$ ). The two-dimensional color map depicts the transmittance versus filter position (vertical axis) and wavelength (horizontal axis). The bandgap and the cavity mode are indicated. Absorption line $(4 \mu \mathrm{m})$ intersects the cavity mode at filter position $12.5 \mathrm{~mm}$. (b) Difference of the intensity integrals as a function of wavelength with and without the analyte versus filter position indicates the intersection point. The difference function is given by Eq. (1). (c) Derivative of the difference signal further highlights the absorption peak.

\section{Fabrication of Variable Photonic Bandgap Filter}

Next, we fabricated the spatially variable IR-PBG filter as shown in Fig. 3. Utilizing the fact that the cavity mode and the PBG scale linearly with the quarter-wave-stack thicknesses, we evaporated 20 layers of $\mathrm{As}_{2} \mathrm{~S}_{3}$ and $\mathrm{Ge}_{15} \mathrm{As}_{25} \mathrm{Se}_{15} \mathrm{Te}_{45}$ with a $40^{\circ}$ slant on a silicon substrate to obtain a positiondependent stop band and a cavity mode. The fabrication process is described in detail in Ref. [1]. The position-dependent PBG and cavity mode are measured using a FTIR microscope (Bruker Vertex 70 Hyperion), as shown in Fig. 4 . The cavity mode shifts from 2.0 to $8.0 \mu \mathrm{m}$ along a $60 \mathrm{~mm}$ long silicon substrate, resulting a $0.1 \mathrm{~nm}$ per micrometer resolution.

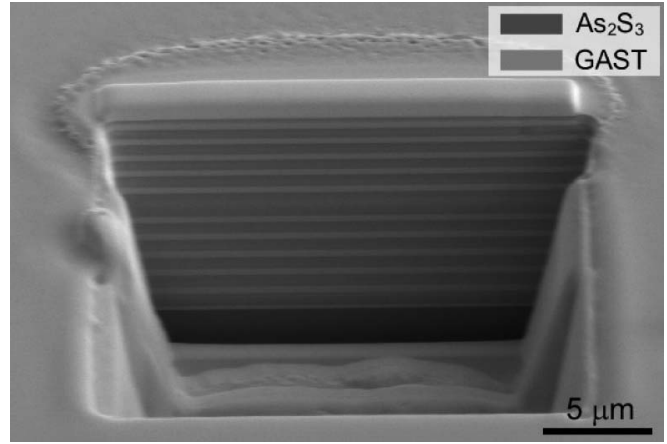

Fig. 3. Cross-sectional scanning electron micrograph of the IR-PBG structure. The structure has 11 pairs of $\mathrm{As}_{2} \mathrm{~S}_{3} /$ $\mathrm{Ge}_{15} \mathrm{As}_{25} \mathrm{Se}_{15} \mathrm{Te}_{45}$ alternating multilayers with a $\mathrm{As}_{2} \mathrm{~S}_{3}$ resonant cavity at the center.

The FWHM of the cavity mode varies from 50 to $80 \mathrm{~nm}$ along the filter length.

\section{Measurements and Results}

The detection performance of the spectrometer is demonstrated using carbon dioxide gas as an analyte; this gas has a pronounced IR absorption peak at $4.3 \mu \mathrm{m}$. We used a FTIR system's blackbody source and detector to measure the total intensity independent of wavelength. The FTIR system's blackbody source and its detector are used in the measurements. However, we emphasize that the detector is only used to obtain a total intensity over a definite wavelength range. The PBG filter is mounted on a movable stage and placed inside the FTIR sample compartment. The incoming beam is passed through pinholes to block the background incident radiation. A background spectrum is obtained for each position of the PBG filter after flushing the sample compartment with nitrogen. Next the chamber is filled with

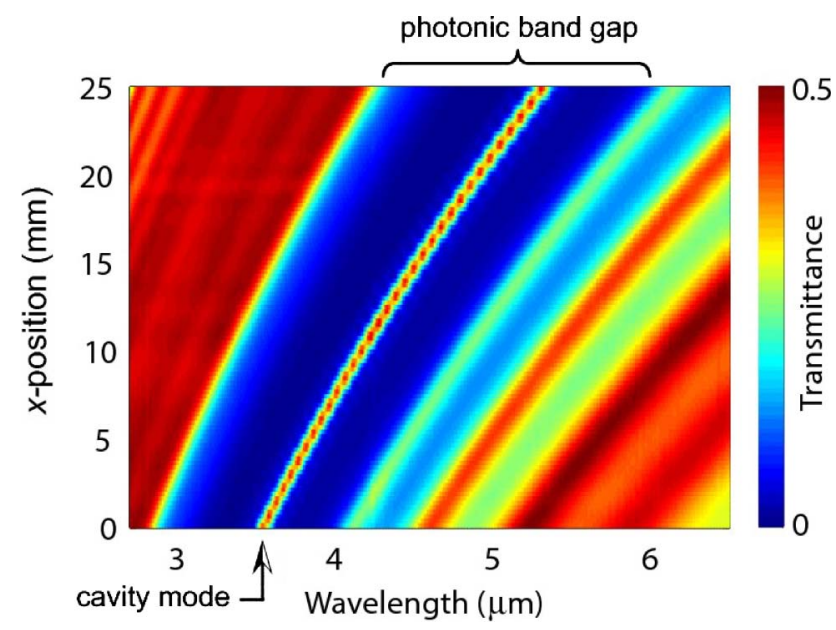

Fig. 4. (Color online) Two-dimensional color map of the fabricated IR-PBG filter depicting the transmittance versus filter position (vertical axis) and wavelength (horizontal axis). The transmittance signal is taken at 40 linearly spaced positions along the $25 \mathrm{~mm}$ part of the filter using an FTIR system. The photonic bandgap and the cavity mode are indicated. 
(a)
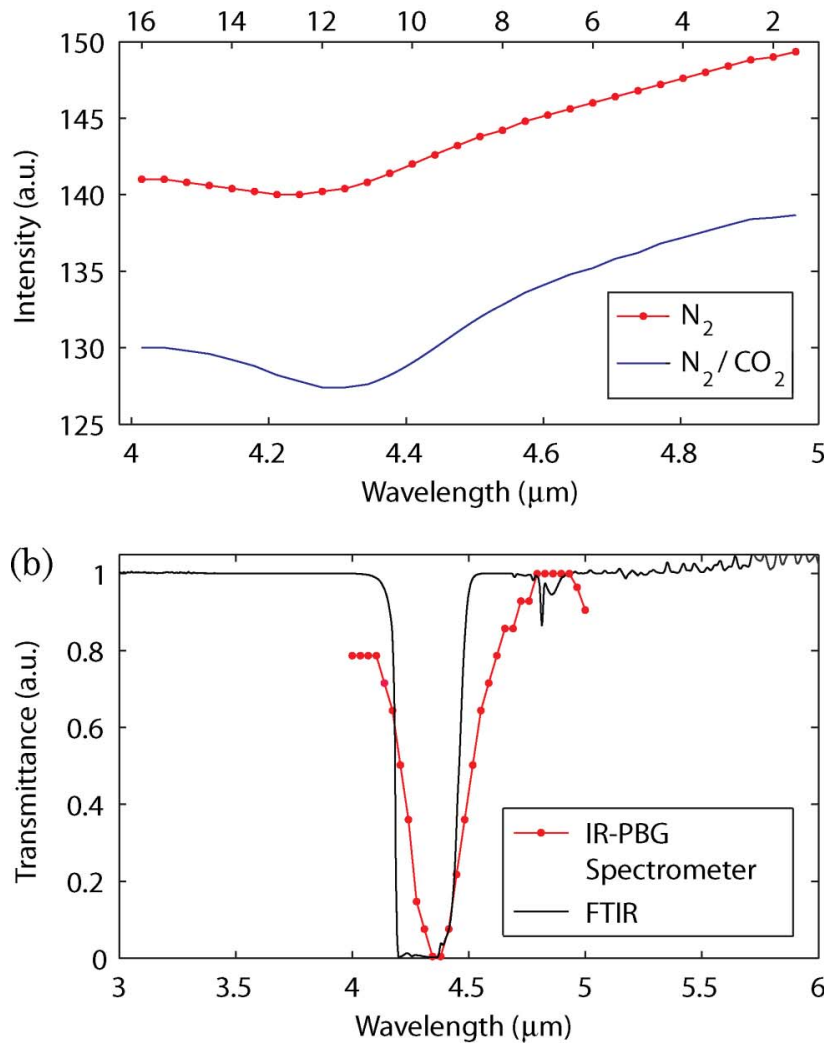

Fig. 5. (Color online) (a) Measured intensity integrals as a function of wavelength with $\left(50 \% \mathrm{CO}_{2}, 50 \% \mathrm{~N}_{2}\right)$ and without (only $\mathrm{N}_{2}$ ) configuration versus filter position. Double $x$ axes represent filter position and corresponding cavity mode wavelength. (b) Difference of the intensity integrals is compared with a typical FTIR absorption spectrum of $\mathrm{CO}_{2}$.

the analyte $\left(\mathrm{CO}_{2}\right)$ and an intensity is obtained for each position of the filter, with $200 \mu \mathrm{m}$ steps, corresponding to a specific wavelength [Fig. 5(a)]. The difference signal reveals the analyte absorption at $4.35 \mu \mathrm{m}$ clearly, as shown in Fig. 5(b). Here, resolution of this measurement is cavity bandwidth limited (50 $\mathrm{nm}$ ) since $200 \mu \mathrm{m}$ steps correspond to $20 \mathrm{~nm}$ cavity mode shift using the $0.1 \mathrm{~nm} / \mu \mathrm{m}$ resolution.

\section{Conclusion}

In the present work we have demonstrated the detection of a gaseous analyte by spatially scanning a variable PBG filter. Furthermore, as discussed in Section 2, a bolometer-array-based design can remove the movable parts. Even though, for imaging purposes, high-sensitivity bolometers are required, in the case of chemical sensing, the light source can be chosen with enough intensity to allow the use of relatively lower detectivity bolometer arrays. Combining our approach with low-cost bolometer arrays, it should be possible to fabricate IR spectrometers with no moving components, making this approach particularly suitable for portable sensing applications.

In addition, an IR-PBG spectrometer features multiple advantages over gas sensors that make use of adsorption or chemical effects. The primary advantage is the lifetime of the device. Typical gas sensors have relatively short lifetimes due to deterioration of the sensor due to environmental exposure. In principle, because no chemical interaction is present in a spectrometer, the lifetime of the sensor will be indefinite. Additionaly, the selectivity of a spectrometer is important in distinguishing multiple components, and this type of sensor is, in principle, able distinguish a large number of gases based on tabulated spectra. Typical bolometers feature 5 to $20 \mathrm{~ms}$ response times, which are limited by the thermal time constants of the device. The bolometer arrays can acquire full spectra within such a time constant. When an intense light source can be used, due to relaxed requirements on detectivity, a reduction of bolometer response time can be achieved by choosing a speedoptimized design. The setup requires minimal alignment, and features no moving parts. Therefore, the system can be particularly suitable in environmental monitoring applications for health or security purposes.

We thank O. Koylu for helping with the experiments. This work is supported by the Scientific and Technological Research Council of Turkey (TUBITAK) under projects 106G090 and 107T547. M. B. acknowledges support from the Turkish Academy of Sciences Distinguished Young Scientist Award (TUBA GEBIP). This work was performed at UNAMInstitute of Materials Science and Nanotechnology, which is supported by the State Planning Organization of Turkey through the National Nanotechnology Research Center Project.

\section{References}

1. H. E. Kondakci, M. Yaman, O. Koylu, A. Dana, and M. Bayindir, "All-chalcogenide glass omnidirectional photonic bandgap variable infrared filters," Appl. Phys. Lett. 94, 111110 (2009).

2. P. Yeh, A. Yariv, and C.-S. Hong, "Electromagnetic propagation in periodic stratified media. I. General theory," J. Opt. Soc. Am. 67, 423-438 (1977).

3. Y. Fink, J. N. Winn, S. H. Fan, C. P. Chen, J. Michel, J. D. Joannopoulos, and E. L. Thomas, "A dielectric omnidirectional reflector," Science 282, 1679-1682 (1998).

4. Z. U. Borisova, Glassy Semiconductors (Plenum, 1981).

5. M. Yaman, H. E. Kondakci, and M. Bayindir, "Large and dynamical tuning of a chalcogenide Fabry-Perot cavity mode by temperature modulation," Opt. Express 18, 3168-3173 (2010).

6. S. D. Hart, G. R. Maskaly, B. Temelkuran, P. H. Prideaux, J. D. Joannopoulos, and Y. Fink, "External reflection from omnidirectional dielectric mirror fiber," Science 296, 510-513 (2002).

7. B. Temelkuran, S. D. Hart, G. Benoit, J. D. Joannopoulos, and Y. Fink, "Wavelength-scalable hollow optical fibres with large photonic bandgaps for $\mathrm{CO}_{2}$ laser transmission," Nature 420, 650-653 (2002).

8. M. Bayindir, F. Sorin, A. F. Abouraddy, J. Viens, S. D. Hart, J. D. Joannopoulos, and Y. Fink, "Metal insulator semiconductor optoelectronic fibres," Nature 431, 826-829 (2004).

9. A. F. Abouraddy, M. Bayindir, G. Benoit, S. D. Hart, K. Kuriki, N. Orf, O. Shapira, F. Sorin, B. Temelkuran, and Y. Fink, "Towards multimaterial multifunctional fibres that see, 
hear, sense and communicate," Nat. Mater. 6, 336-347 (2007).

10. C. Charlton, B. Temelkuran, G. Dellemann, and B. Mizaikoff, "Midinfrared sensors meet nanotechnology: trace gas sensing with quantum cascade lasers inside photonic band-gap hollow waveguides," Appl. Phys. Lett. 86, 194102 (2005).
11. P. Domachuk, I. C. M. Litter, M. Cronin-Golomb, and B. J. Eggleton, "Compact resonant integrated microfluidic refractometer," Appl. Phys. Lett. 88, 093513 (2006).

12. A. Jahanzeb, C. M. Travers, Z. Celik-Butler, D. P. Butler, and S. G. Tan, "A semiconductor $\mathrm{YBaCuO}$ microbolometer for room temperature IR imaging," IEEE Trans. Electron. Devices 44, 1795-1801 (1997). 